\title{
Percepciones sobre la diversidad sexual en adolescentes escolarizados de la ciudad de Cuenca
}

\author{
Verónica P. Pinos V. ${ }^{1}$, Guido M. Pinos A. ${ }^{2}$, María D. Palacios $M^{3}$ \\ ${ }^{1}$ Dirección de Investigación de la Universidad de Cuenca (DIUC), Cuenca, Ecuador \\ ${ }^{2}$ Facultad de Ciencias Médicas, Universidad de Cuenca, Cuenca, Ecuador \\ ${ }^{3}$ Facultad de Psicología, Universidad de Cuenca, Cuenca, Ecuador \\ Autor para correspondencia: veronica.pinos@ucuenca.edu.ec \\ Fecha de recepción: 1 de enero de 2012 - Fecha de aceptación: 20 de febrero de 2012
}

\begin{abstract}
RESUMEN
Lesbianas, gays, bisexuales y transexuales (LGBT) son un grupo minoritario relevante en la comunidad, cuyo reconocimiento despierta reacciones emocionales, percepciones y actitudes, la mayoría de tendencia negativa. Las percepciones y actitudes de rechazo se deben en gran parte a los prejuicios contra ese grupo minoritario, que a menudo conduce a una estigmatización de las personas LGBT. El propósito principal de la investigación fue la evaluación de las percepciones de los adolescentes hacia las personas LGBT. Una encuesta, que consta de una serie de preguntas cualitativas y cuantitativas que ofrece a los encuestados la posibilidad de proporcionar comentarios y observaciones personales, se aplicó a 1130 estudiantes y a 31 de sus profesores de colegio. Los estudiantes, en general, señalaron que no es correcto maltratar a las personas LGBT. Además, ellos percibieron que la familia, la escuela y sobre todo la sociedad son ambientes hostiles para LGBT. Por otra parte, la mayoría expresaron que en el supuesto caso de ser ellos LGBT, no sabrían la reacción de su familia, amigos, profesores y pares; incluso percibieron rechazo por parte de sus amigos y compañeros, presión para que cambien de parte de sus padres, y una actitud de indiferencia por parte de sus profesores, al considerar que pocas veces se preocupan por la situación personal de los estudiantes; además, teniendo en cuenta la opinión pública generalizada y considerando que usualmente son blanco de fuertes actitudes negativas, algunos adolescentes dijeron que probablemente no lo harían público. En conclusión, para lograr la equidad social para las personas LGBT se debe trabajar por la liberación de perjuicios sexuales en la sociedad en general, lo que requiere el inicio de una serie de actividades, entre ellas, la iniciación de programas educativos con el objetivo de eliminar prejuicios y promover mentes abiertas a las diferencias sexuales.
\end{abstract}

Palabras clave: Diversidad sexual, percepciones, actitudes, LGBT, lesbianas, homosexuales, bisexuales, transexuales, adolescentes, homofobia.

\begin{abstract}
Lesbian, gay, bisexual and transgender (LGBT) people are a minority group, whose recognition awakens emotional reactions, perceptions and attitudes; the majority with negative tendence. The perceptions and attitudes of rejection are due in large part to the prejudice against this group, which often leads to a stigmatization of LGBT people. Assessment of the perceptions of adolescents towards LGBT individuals was the main purpose of the research. A survey, consisting of a series of qualitative and quantitative questions offering the surveyed people the possibility of providing to the questions personal comments and observations, was applied to 1130 college students and 31 teachers. The students in general stated that it is incorrect mistreating LGBT people. They strongly perceive that family, school and especially the society as hostile environments for LGBT. Moreover, most of the surveyed adolescents expressed serious concern that if they were LGBT, they would worry about the emotional reaction of family, friends, teachers and peers. In the worst case, they even expect being rejected by friends and peers, experience pressure from their family to change attitude, and likely be
\end{abstract}


confronted by an indifferent attitude of their teachers as the latter usually seldom care about the personal situation of students. Given the widespread public opinion and the risk to become the target of strong negative attitudes some adolescents stated to be very reluctant to make public their sexual orientation. It is believed that for achieving social equity for LGBT people one has to work towards the liberation for the broader society from sexual oppression, requiring the initiation of a series of activities, such as the initiation of educational programs aiming de-ligitimization of the prejudice and the opening of people's mind.

Keywords: Sexual diversity, perceptions, attitudes, LGBT, lesbians, gays, bisexuals, transsexuals, adolescents, homophobia.

\section{INTRODUCCIÓN}

La identidad de género es la percepción psicológica de pertenecer a un sexo y habitualmente coincide con el sexo de nacimiento. Esta incluye dos componentes, los cuales ya se manifiestan en los primeros años de vida: el primero, es el reconocimiento biológico de ser mujer o varón y el segundo es el aprendizaje social de las expectativas de feminidad y masculinidad dentro de la sociedad. Cuando la identidad de género es contraria al sexo biológico estamos hablando de transexualidad (T). La orientación sexual hace referencia a la preferencia que tiene un sujeto en sus relaciones afectivas o eróticas. Entre las orientaciones sexuales podemos mencionar a la homosexualidad, que es la preferencia hacia sujetos del mismo sexo; la bisexualidad, que es la atracción erótica por sujetos de ambos sexos; la heterosexualidad, que es la preferencia por sujetos del sexo opuesto; y la asexualidad, que es la ausencia de atracción por el sexo (Crooks y Baur, 2009).

Las etapas para que el individuo LGB (lesbiana, gay y bisexual) asuma su orientación sexual son: sensibilización, donde el niño o adolescente se siente diferente; conciencia con confusión, habitualmente viene acompañada de negación; culpa y vergüenza; y finalmente, aceptación de identidad (Ardila, 2002; Crooks y Baur, 2009; Shibley y Delamater, 2006). Con respecto a la conciencia con confusión, si bien, algunos individuos atraviesan este proceso con gran sufrimiento y confusión, afectando su desarrollo afectivo, otros pasan este proceso rápidamente y sin ninguna complicación (Whitlock, 1989). Como generalidad, la conciencia de atracción por el mismo sexo se inicia entre $\operatorname{los} 8$ y 11 años, los comportamientos sexuales ocurren entre los 12-15 años, la identificación como homosexuales se da entre los 15-18 años, la revelación a los demás se produce entre los 17-19 años, finalmente el desarrollo de relaciones románticas con personas del mismo sexo se establece entre los 18-20 años (Papalia y col., 2005).

Una posible explicación para que muchos adolescentes LGBT tarden en mostrar su verdad o la oculten ante su familia, sus pares y en general su entorno, es debido a que esperan una respuesta de rechazo y negación por parte de quienes les rodean; lamentablemente, la realidad muestra que estos miedos y temores son fundamentados (González y col., 2004). Esta angustia y miedo hacia los demás, además del auto-rechazo que muchos LGBT sienten a su orientación sexual se puede explicar por la relación que existe entre la interacción social y el autoconcepto. Somos concientes de quienes somos a través de nuestras experiencias en el mundo, y de nuestras interacciones con otras personas. Las opiniones de las personas nos influyen en cualquier aspecto y tema, el autoconcepto no es la excepción; a esto se lo conoce como valoración reflejada, pues en nuestra autoevaluación se manifiesta la percepción que los demás tienen de nosotros. En niños y adolescentes cobra especial importancia, ya que para ellos lo que piensan sus padres y otros seres queridos o lo que imaginan que estos piensan, afecta no solo a su autoconcepción sino además a su sentimiento de valía (Worchel y col., 2003).

En general, se percibe lo que las otras personas piensan mediante la sistematización de la cognición social (Martínez y Sampedro, 2003). Llamamos cognición social al proceso que persigue entender a las otras personas; sus componentes fundamentales son las atribuciones sobre la razón por la cual la gente actúa de determinada manera y la formación de una impresión general en función de lo que sabemos o creemos saber de un determinado objeto social (Worchel y col., 2003). La cognición 
puede ser calificada como social desde tres perspectivas: por su origen (es creada y reforzada a través de la interacción social), el objeto evaluado es social (grupos, personas, relaciones, etc.) y finalmente es socialmente compartida entre los diferentes grupos y miembros de la sociedad (Martínez y Sampedro, 2003).

Aunque en general compartimos con las personas la información que nos proporciona el medio social, no todos construimos el conocimiento de la misma manera; es decir, nuestra percepción social no solo va a depender de las características materiales u objetivas de lo que percibimos, sino además de nuestra construcción activa de la realidad (Martínez y Sampedro, 2003). La percepción es el proceso por el cual seleccionamos, organizamos e interpretamos la entrada a nuestros receptores sensoriales (Barón, 1997). La percepción social es la apreciación que un individuo hace de las personas, de su entorno social y el juicio que les atribuye; presenta las siguientes fases: interpretación de indicios (la selección y codificación de los datos del exterior), formación de impresiones, elaboración de inferencias (atribuciones o búsquedas de las posibles causas de los acontecimientos que observamos) y los sesgos inevitables debidos a idiosincrasia de cada individuo (Martínez y Sampedro, 2003). Por cuanto, las percepciones sociales o interpretaciones sobre lo que las otras personas piensan sobre un determinado objeto social, influye en las personas, en su manera de verse a sí mismos y además les da una idea de lo que tendrán que enfrentar o lo que les espera en una situación dada.

En el caso particular de los LGBT: ¿Cuál es el medio que les espera? ¿Qué es lo que perciben con respecto a su entorno? Diferentes estudios han detectado dos tendencias de reacción hacia LGBT; la dirección negativa que se caracteriza por los componentes: cognitivo (es anormal, son peligrosos, son raros), afectivo (ansiedad, incomodidad, malestar, asco, rechazo) y conductual (evitación, mofa, agresión) y la dirección positiva que se caracteriza por los componentes: cognitivo (considera a los homosexuales igual que los demás), afectivo (aceptación) y conductual (convivencia normalizada) (Soriano, 1995). Lesbianas y gays enfrentan diversas formas de exclusión social debido a que en la mayoría de las sociedades se valora de forma negativa su orientación sexual, considerándolos un grupo homogéneo de personas con características negativas; según esta visión son enfermos, delincuentes, pecadores y carentes de moral (Ortiz, 2005). A nivel local, se ha encontrado que tanto adolescentes cuanto profesores mantienen creencias prejuiciosas en contra de los LGBT, las cuales se relacionan con actitudes homofóbicas (Pinos y Pinos, 2011). Estos estereotipos, valores e ideologías emergentes de nuestro contexto cultural, generan en los LGBT marcas psicológicas y sociales, que se conocen como estigmas. El estigma es un término que implica tanto la desviación como el prejuicio y va más allá de ambos, es una condición, atributo, rasgo o comportamiento que hace que su portador sea incluido en una categoría social, cuyos miembros provocan una respuesta negativa (Dovido y col., 2003). Los individuos que portan algún estigma presentan dos etapas en su socialización: la primera, cuando se adquieren las creencias sobre el estigma y una idea general de lo que significa portarlo; y la segunda, cuando se autoidentifican como portadores de este (Goffman, 1995). Los LGBT son conscientes de ser estigmatizados o perciben su estigma, reconocen que sus personas cercanas pueden ser influidas por dichos estereotipos e inconscientemente, presionarlos a actuar en relación a estas expectativas; finalmente, saben que las reacciones y las actitudes de las demás personas hacia ellos pueden depender de los estereotipos que tengan (Ortiz, 2005).

Son muchos los indicios que llevarían a pensar que pese a los esfuerzos mundiales que buscan el respeto a la diversidad sexual, aun existe discriminación hacia esta minoría, provocando perjuicio a su integridad emocional y psicológica (Santrock, 2004; Shibley y Delamater, 2006). De hecho, se ha encontrado autohomofobia en adolescentes LGBT debido a la estigmatización que perciben (Ortiz, 2005); además, frecuentemente usan mecanismos de defensa para protegerse de esta estigmatización como la negación y la racionalización en el caso de haber tenido conductas homosexuales (Santrock, 2004). Asimismo, cuando un individuo reconoce su orientación sexual, casi nunca son los padres o las madres las primeras personas en saberlo, y entre estos, son usualmente las madres las que conocen primero dicha situación (Bertone y Franchi, 2008; Santrock, 2004). De otra parte, entre el 50\% y 60\% de los adolescentes LGBT confiaron en un hermano para contarles sobre su orientación sexual aunque generalmente es un amigo la primera persona en saberlo; sin embargo, muchos LGBT, para evitar la estigmatización se "esconden dentro del armario", es decir, ocultan su orientación hasta el momento de sentirse seguros, que es generalmente en la universidad (Santrock, 2004). 
Investigaciones realizadas en Italia han encontrado que hay quienes ocultan su orientación de por vida, o se mantienen semi-visibles, compartiendo esta información de mayor a menor grado con sus amistades, familia y compañeros de colegio o trabajo (Graglia y col., 2008). Trabajos realizados en Bolivia, Italia y Chile descubrieron que entre los padres y madres se pueden encontrar diferentes reacciones al enterarse de la orientación sexual del hijo/a, que van desde el apoyo incondicional hasta la presentación de conflictos dentro de la familia (Antezana, 2007; Bertone y Franchi, 2008; González y col., 2004). Un estudio Italiano estableció entre estas reacciones generadas, casos de petición para que él/ella oculte su orientación sexual, o busque una cura, además de casos donde los hijos(as) recibieron castigo físico, y/o fueron expulsados del hogar (Bertone y Franchi, 2008). Por otra parte, un estudio mexicano, encontró una relación entre la homofobia internalizada, la percepción del estigma por su homosexualidad, el ocultamiento, con un mayor riesgo de presentar ideación suicida, intento de suicidio, trastornos mentales y alcoholismo (Ortiz, 2005).

Un estudio realizado en España, en adolescentes, encontró que el $6 \%$ de encuestados consideró correcto violentar a un LGBT y un 7,4\% lo justificarían según el caso. Los participantes consideraron que en la familia LGBT son tratados: injustamente el 32,8\%, como los demás el 59,9\% y más favorablemente el 3,7\%; en el colegio: de manera más injusta el 76,2\%, como a los demás el 19,2\% y de forma más favorable el 1,4\%; en la sociedad: de manera más injusta un 72,6\%, como los demás el $21 \%$ y más favorablemente en el 3,4\%. Además, de ser ellos mismo LGBT esperarían el siguiente trato, en la familia: maltrato físico el 3,6\%, rechazo el $7 \%$, indiferencia ante el tema el $16,6 \%$, no sabrían cómo ellos reaccionarían el 38,5\%, apoyo el 53,2\% e intentar cambiar su orientación el 19\%; con los amigos: maltrato físico el 5,8\%, rechazo el 24,9\%, indiferencia el 14,3\%, no saben el 38,5\%, apoyo el 31,7\% e intentar cambiar su orientación el 19,3\%; en los profesores: maltrato físico el 1,7\%, rechazo el 3,3\%, indiferencia el 35,5\%, no saben el 32,3\%, apoyo el $25,1 \%$ e intentar cambiar su orientación el 4,7\%; con sus compañeros: maltrato físico el 13,4\%, rechazo el 41,7\%, indiferencia el 10,9\%, no saben el 37,6\%, apoyo el 8,4\% e intentar cambiar su orientación el 15,3\% (Pichardo y col., 2009a\&b).

Por todo lo antes mencionado, nuestro objetivo fue el conocer las percepciones de los adolescentes hacia la comunidad LGBT en sus espacios vitales. Este conocimiento nos servirá como una base para llegar de una manera más adecuada a sensibilizarlos en este tema de interés humano mediante programas educativos.

\section{METODOLOGÍA}

Para la realización de esta investigación se aplicó un enfoque mixto multimodal de un solo paso, con este propósito se empleó un cuestionario de preguntas cerradas para la obtención de los datos cuantitativos y otras abiertas para obtener datos cualitativos (Creswell, 2008; Hernández y col., 2007). El universo de este estudio lo constituyeron los estudiantes adolescentes escolarizados en secundaria de la ciudad de Cuenca, el marco muestral estuvo conformado por los colegios de la ciudad. El muestreo fue probabilístico por racimos o clústeres (Hernández y col., 2007). Se sortearon los colegios, y a continuación los paralelos, desde el octavo de básica hasta tercero de bachillerato. Un total de 1130 estudiantes y 31 de sus profesores fueron encuestados de manera voluntaria.

Para la recolección de los datos, se aplicó el cuestionario español de actitudes hacia la diversidad sexual (Pichardo y col., 2009a\&b). A este, se le incluyó, en las secciones: actitudes hacia el maltrato de LGBT, percepciones sobre el trato de LGBT en sus espacios vitales (familia, colegio y sociedad) y percepciones de la reacción del otro (familia, amigos, compañeros y maestros) de ser ellos LGBTy la pregunta ¿por qué?, con el fin, de que se explique a profundidad la razón de la elección de la opción cuantitativa. Se calcularon las frecuencias y porcentajes de los datos cuantitativos y se realizó análisis del contenido en los datos cualitativos, para este análisis se realizó la selección de los contenidos de la categorización, de las unidades de análisis y del sistema de recuento o medida (Piñuel, 2002). Cada segmento de frase fue analizado y categorizado, se eliminaron las respuestas que no correspondían con la pregunta. La triangulación fue realizada mediante la fuente de información, ya que se tuvo una 
extensa base de datos, con las respuestas de la encuesta aplicada. El análisis fue realizado por tres miembros del equipo de investigación.

Entre los posibles sesgos se tiene que un porcentaje importante de encuestados no respondieron las preguntas abiertas; además, algunas respuestas no eran pertinentes, pues no se relacionaban con la pregunta realizada, así que no fueron consideradas en el análisis cualitativo. Otro sesgo a considerar es la amplitud de las preguntas pues consideran LGBT como una unidad. Otro aspecto es que los instrumentos utilizados para evaluar estas actitudes han sido cuestionarios y escalas; sin embargo, estos instrumentos de evaluación han sido criticados debido a ciertas limitaciones detectadas en ellos, tales como: la diferente capacidad de las personas de ser conscientes de sus propias actitudes y estados internos; la deseabilidad social de las respuestas; la presentación positiva que intentan hacer de sí mismos en vistas a preservar una auto-imagen positiva; entre otros (Briñol, 2001).

\section{RESULTADOS Y DISCUSIÓN}

Los 1130 adolescentes encuestados se distribuyeron de la siguiente manera: 531 (47\%) pertenecían a un colegio fiscal y $599(53 \%)$ a un colegio particular de la ciudad de Cuenca; $482(42,65 \%)$ son mujeres y $634(56,11 \%)$ varones; $307(27,17 \%)$ tenían entre 11 a 13 años (adolescentes tempranos), $583(51,59 \%)$ estaban entre 14 y 16 años (adolescentes medios) y $231(2,44 \%)$ tenían entre 17 y 19 años (adolescentes tardíos). Los 31 profesores encuestados se distribuyeron según su lugar de trabajo, de la siguiente manera: $11(35,48 \%)$ trabajaban en colegios fiscales y $20(64,52 \%)$ en colegios particulares. Según la edad: $9(29,03 \%)$ tenían entre 21 y 30 años, $10(32,26 \%)$ estaban entre los 31 a 40 años, $6(19,35 \%)$ entre los 41 y 50 años, $5(16,13 \%)$ entre 51 y 60 años, $1(3,23 \%)$ entre 61 y 70 años.

\subsection{Actitudes hacia el maltrato a los LGBT}

Los adolescentes consideraron en su mayoría que se debe respetar a las personas que tienen orientación LGBT, aunque un preocupante 21,68\% indicó que a veces justificarían la agresión, según el caso. La tendencia encontrada en esta pregunta coincide con la encontrada en el estudio realizado en España (Pichardo y col., 2009a\&b).Donde, el 67,35\% de los participantes reconocen que no es correcto tratar con desprecio a LGBT, pues se rescata que ellos son personas que merecen respeto; no obstante, se encontró que prevalece la creencia errónea de que la orientación sexual es una elección y que no es normal. Un grupo de adolescentes alegó que depende del caso, idea muy peligrosa porque incluye la concepción de que la violencia puede ser merecida; se encontró como justificativos para este desprecio: el que ellos se muestren públicamente, que intenten conquistarles o sean agresivos; mientras que, los factores que favorecerían la aceptación serían: el tener conocidos en este grupo y que tengan la intención de cambiar. Finalmente, al examinar las razones para este menosprecio fueron expresadas las siguientes: el considerar que no es normal, que producen asco y que son peligrosos; razones forman parte de los componentes cognitivo y afectivo, de las tendencias negativas hacia esta minoría, como lo afirma (Soriano, 1995). Todo esto indica que aún se mantienen prejuicios hacia esta minoría, los que son el soporte para su estigmatización, como lo indica (Ortiz, 2005).

Por otra parte, las adolescentes lesbianas, $11(78,57 \%)$ respondieron que el desprecio no es correcto pues "Somos personas con derechos", "todos tienen derecho a ser felices", 1 (7,14\%) que si es correcto "Porque Dios hizo a la mujer y al hombre para que se complementen entre ellos, no hizo solo hombres o solo mujeres para que se junten entre ellos es decir personas del mismo sexo" y 3 $(21,43 \%)$ manifestaron que depende del caso. Entre los adolescentes gay: 7 (50\%) respondieron que no es correcto, $1(7,14 \%)$ que si es correcto y $6(42,86 \%)$ que depende del caso "..si fuera un abusador que le gusta del mismo sexo". Entre las y los adolescentes bisexuales 27 (90\%) respondieron que no es correcto, "Porque todos tenemos una libertad absoluta...todos somos iguales, no podemos discriminar solo por un gusto sexual, es el mismo amor y cariño que se da en una pareja heterosexual, estoy de acuerdo con el lesbianismo, bisexuales, homosexuales, etc., y les apoyo totalmente", y $4(13,33 \%)$ que depende del caso "Hay personas que violan a los de su mismo sexo". 
Tabla 1. ¿Crees que es correcto tratar con desprecio a personas a las que les gustan los de su mismo sexo?

\begin{tabular}{|c|c|c|c|c|c|c|}
\hline & $\mathrm{n}$ & $\%$ & ¿Por qué? & $\mathrm{N}$ & $\%$ & Citas \\
\hline \multirow{3}{*}{$\begin{array}{l}\stackrel{0}{0} \\
\stackrel{0}{0} \\
\text { ర0 } \\
0 \\
0 \\
0 \\
z\end{array}$} & \multirow{3}{*}{761} & \multirow{3}{*}{67,35} & Son personas & 526 & 69,12 & $\begin{array}{l}\text { “...son personas, tienen sentimientos y al } \\
\text { igual que yo, sienten amor hacia otra } \\
\text { persona que bien puede ser hombre o mujer, } \\
\text { lo importante es amar" }\end{array}$ \\
\hline & & & Es su decisión & 139 & 18,27 & $\begin{array}{l}\text { "Las personas se encuentran con libertad } \\
\text { para elegir su orientación sexual..." }\end{array}$ \\
\hline & & & $\begin{array}{l}\text { Pero no es } \\
\text { normal }\end{array}$ & 68 & 8,94 & $\begin{array}{l}\text { "...el hecho que para mi este mal eso y me } \\
\text { da asco, no quiere decir que no respetemos } \\
\text { sus derechos" }\end{array}$ \\
\hline \multirow{3}{*}{$\begin{array}{l}\stackrel{0}{0} \\
\stackrel{0}{0} \\
0 \\
0 \\
0 \\
i n\end{array}$} & \multirow{3}{*}{32} & \multirow{3}{*}{2,83} & No es normal & 9 & 28,13 & $\begin{array}{l}\text { "...no debería haber personas gay, porque } \\
\text { dios hizo al hombre para que tenga hijitos } \\
\text { con una mujer" }\end{array}$ \\
\hline & & & Producen asco & 4 & 12,50 & "Me da asco ver dos hombres besándose" \\
\hline & & & Son agresivos & 2 & 6,25 & “...pueden venir contra uno mismo" \\
\hline \multirow{6}{*}{ 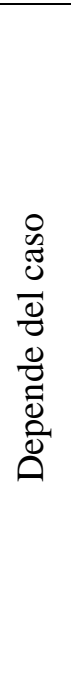 } & \multirow{6}{*}{245} & \multirow{6}{*}{21,68} & Según la situación & 42 & 17,14 & $\begin{array}{l}\text { “...dependería mucho de cuál es esa } \\
\text { circunstancia para saber cómo llevarla y } \\
\text { como tratar a esa persona” }\end{array}$ \\
\hline & & & Si se exhiben & 32 & 13,06 & $\begin{array}{l}\text { "Depende si actúan en público, si es que es } \\
\text { gay pero no está haciendo notar mucho, no } \\
\text { importa" }\end{array}$ \\
\hline & & & Si se insinúan & 21 & 8,57 & $\begin{array}{l}\text { “... veces esa persona puede intentar algo } \\
\text { con vos y ahi tienes que ponerle las cosas } \\
\text { claras" }\end{array}$ \\
\hline & & & Si son conocidos & 18 & 7,35 & $\begin{array}{l}\text { "...si es conocido no puedes decir nada pero } \\
\text { si no lo es, si" }\end{array}$ \\
\hline & & & Si son agresivos & 14 & 5,71 & $\begin{array}{l}\text { "...nos pueden hacer daño es decir acaricie } \\
\text { a la fuerza y tú no quieras" }\end{array}$ \\
\hline & & & Si cambian & 5 & 2,04 & “...tal vez reflexionen y se hagan normales" \\
\hline
\end{tabular}

En cuanto a sus profesores $25(80,65 \%)$ respondieron que no está bien agredir a las personas LGBT, aunque en algunos casos fue considerado un pecado "Porque la homosexualidad no se considera ni se ha considerado normal bajo ningún punto de vista, Dios creó hombre y mujer como pareja y para que así se multiplicaran". 3 (9,68\%) indicaron que dependería del caso, "Porque hay personas que demuestran respeto pero algunos no tienen ni el mínimo respeto por nada" y 1 (3,23\%) consideró que sí porque "es una falta de respeto". Como se puede ver, tanto los adolescentes LGB cuanto sus profesores, siguieron la tendencia general del grupo en sus respuestas; rechazando la discriminación hacia ellos, pese a que algunos mantienen prejuicios que justifican la agresión.

\subsection{Percepciones del trato hacia los LGBT}

En la familia

Los adolescentes encuestados estuvieron divididos en sus percepciones del trato a los LGBT dentro del núcleo familiar, pues aunque un $42,92 \%$ consideraron que no son injustamente tratados, un porcentaje similar, $40,97 \%$, indicó que no existe una diferencia de trato. Los resultados encontrados difieren en tendencia con los del estudio de España, pues la mayoría de adolescentes españoles expusieron que los LGBT son tratados como los demás dentro del núcleo familiar (Pichardo y col., 
2009a\&b). Esto puede deberse a que en España este tema se trata más abiertamente y desde hace más tiempo que en nuestro país.

Tabla 2. ¿Cómo crees que se trata a LGBT en la familia, en comparación con los otros?

\begin{tabular}{|c|c|c|c|c|c|c|}
\hline & $\mathrm{n}$ & $\%$ & ¿Por qué? & $\mathrm{n}$ & $\%$ & Citas \\
\hline \multirow{6}{*}{ 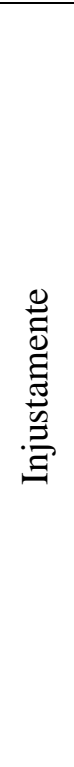 } & \multirow{6}{*}{485} & \multirow{6}{*}{42,92} & Discriminados & 268 & 55,26 & $\begin{array}{l}\text { "...hay muchas personas que no aceptan el hecho } \\
\text { de que un familiar sea así y muchas veces les } \\
\text { discriminan" }\end{array}$ \\
\hline & & & Violentados & 56 & 11,55 & $\begin{array}{l}\text { "...la mayoría los maltratan e insultan por su } \\
\text { orientación sexual y les ven como algo diferente a } \\
\text { la sociedad" }\end{array}$ \\
\hline & & & $\begin{array}{l}\text { Considerado } \\
\text { anormal }\end{array}$ & 54 & 11,13 & $\begin{array}{l}\text { "...la mayoría de las familias los ven como algo } \\
\text { anormal" }\end{array}$ \\
\hline & & & $\begin{array}{l}\text { Considerado } \\
\text { pecado }\end{array}$ & 20 & 4,12 & $\begin{array}{l}\text { "A los padres les parece un pecado muy grande } \\
\text { que sus hijos sean de otro sexo y sentirían } \\
\text { vergüenza de él o ella" }\end{array}$ \\
\hline & & & $\begin{array}{l}\text { Tratan de } \\
\text { cambiarlos }\end{array}$ & 11 & 2,27 & $\begin{array}{l}\text { "De una forma injusta porque en algunos casos } \\
\text { que he visto las familias tratan de que algún } \\
\text { familiar cambie su orientación sexual" }\end{array}$ \\
\hline & & & $\begin{array}{l}\text { Considerado } \\
\text { enfermedad }\end{array}$ & 10 & 2,06 & $\begin{array}{l}\text { "...todavía se cree que es una enfermedad o una } \\
\text { etapa..." }\end{array}$ \\
\hline \multirow{4}{*}{ 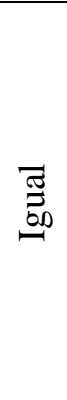 } & \multirow{4}{*}{463} & \multirow{4}{*}{40,97} & Otro familiar & 370 & 79,91 & $\begin{array}{l}\text { "Sí, porque seamos como seamos todos somos } \\
\text { respetados y queridos por nuestros familiares y } \\
\text { ellos no son la excepción" }\end{array}$ \\
\hline & & & $\begin{array}{l}\text { Necesitan } \\
\text { apoyo }\end{array}$ & 12 & 2,59 & $\begin{array}{l}\text { "Ya que sin el apoyo de su familia, ellos podrian } \\
\text { llegar a cometer cosas como matarse" }\end{array}$ \\
\hline & & & Con reservas & 4 & 0,86 & "Pero con un poco de recelo" \\
\hline & & & No lo saben & 3 & 0,65 & $\begin{array}{l}\text { "...normalmente no cuentan a casi nadie su } \\
\text { preferencia sexual" }\end{array}$ \\
\hline \multirow{2}{*}{$\frac{\grave{0}}{\sum^{2}}$} & \multirow{2}{*}{58} & \multirow{2}{*}{5,13} & $\begin{array}{l}\text { Necesitan } \\
\text { apoyo }\end{array}$ & 24 & 41,38 & $\begin{array}{l}\text { "... los familiares tratan de hacerle sentir bien y } \\
\text { no separarlo del grupo" }\end{array}$ \\
\hline & & & $\begin{array}{l}\text { Considerado } \\
\text { enfermedad }\end{array}$ & 4 & 6,90 & $\begin{array}{l}\text { "...teniendo en cuenta que es una enfermedad se } \\
\text { trata a la gente con más atención y cariño" }\end{array}$ \\
\hline
\end{tabular}

Las razones que los adolescentes dieron para sus respuestas fueron: que los LGBT son tratados de manera injusta en sus hogares pues consideran que ellos reciben discriminación, violencia, además de ser catalogados como anormales, pecadores, enfermos y reciben presión para cambiar. Otro grupo consideró que son tratados como al resto de la familia, pues como parte de la familia merecen respeto y apoyo; aunque tambien se expuso la idea, que se mantendría oculta la situación ante ellos. Finalmente, los adolescentes que indicaron que se les trata mejor que al resto, alegaron que la familia siente la necesidad de brindarles más apoyo.

Las adolescentes lesbianas respondieron a esta pregunta, 7 (50\%) de manera injusta "Porque siempre son discriminados", $5(35,71 \%)$ de igual manera que al resto y $1(7,14 \%)$ de manera más favorable. Los adolescentes gays respondieron a esta pregunta, 4 (28,57\%) de manera injusta "Porque a la mayoría se les maltrata", $6(42,57 \%)$ de igual manera que al resto y $4(28,57 \%)$ de manera más favorable. Los y las adolescentes bisexuales respondieron 19 (63,33\%) de manera injusta "Porque piensan que ellos son enfermos, pecadores y que dañan a la sociedad", $12(40 \%)$ de igual manera que al resto "Tenemos los mismos derechos". Los profesores opinaron, $16(51,61 \%)$ de manera más injusta, "Porque por lo general en nuestro país la homofobia, la discriminación a los gays, el aislamiento, la no aceptación de su condición y son injustamente tratados", "Muchas familias los 
consideran como una enfermedad y tratan de curarlo", 11 (35,48\%) como a los demás, "Porque igual son seres humanos", $3(9,68 \%)$ de manera más favorable "Es como si considerara que requiere ayuda". Adolescentes LGB y profesores y el resto de participantes opinaron siguiendo la misma tendencia.

\section{En el colegio}

Un preocupante $60,71 \%$ de los adolescentes encuestados indicaron que perciben que los LGBT son tratados injustamente en el Colegio, y el 2,39\% consideró que son tratados mejor; estos hallazgos coinciden en tendencia con la distribución encontrada en España (Pichardo y col., 2009a\&b). Entre las razones para alegar la percepción de este trato injusto se mencionó que los LGBT son violentados, excluidos, incomprendidos, considerados anormales, pecadores, enfermos, sin derechos y que dan mal ejemplo; pero además, se halló que algunos adolescentes consideraban que esa situación produce asco, miedo, incomodidad, que se los consideran sin derechos y que esta agresión se produce como un intento de hacerlos cambiar. Los que alegaron que son considerados como los demás, indicaron que a la final son compañeros y estudiantes como el resto. Además, se encontró que muchas veces su orientación se mantiene oculta; se resaltó la idea de que hay que apoyarlos y que hoy en día son más aceptados. Los adolescentes que consideraron que son mejor tratados indicaron que esto se debe a que existe la idea de tenerlos que proteger porque son más vulnerables que el resto, otros consideran que reciben apoyos extras.

Tabla 3. ¿Cómo crees que se trata a LGBT en el colegio, en comparación con los otros?

\begin{tabular}{|c|c|c|c|c|c|c|}
\hline & $\mathrm{n}$ & $\%$ & ¿Por qué? & $\mathrm{n}$ & $\%$ & Citas \\
\hline \multirow{13}{*}{ 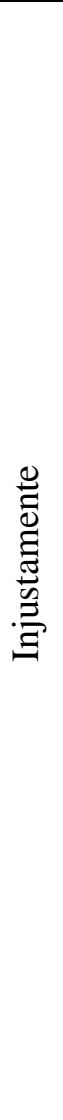 } & \multirow{13}{*}{686} & \multirow{13}{*}{60,71} & Violentados & 315 & 45,92 & $\begin{array}{l}\text { "... estas personas reciben muchos } \\
\text { abusos" }\end{array}$ \\
\hline & & & Excluidos & 101 & 14,72 & $\begin{array}{l}\text { "Casi todos los del colegio discriminan } \\
\ldots .\end{array}$ \\
\hline & & & No aceptados & 66 & 9.62 & "No...tienen una buena aceptación..." \\
\hline & & & $\begin{array}{l}\text { Considerado } \\
\text { anormal }\end{array}$ & 58 & 8,45 & “... para algunos eso no es normal..." \\
\hline & & & $\begin{array}{l}\text { Considerado } \\
\text { pecado }\end{array}$ & 16 & 2,33 & $\begin{array}{l}\text { "... tienen una creencia...que eso está mal, } \\
\text { que es pecado" }\end{array}$ \\
\hline & & & Producen asco & 13 & 1,90 & "...tiene... asco al ver a uno de ellos" \\
\hline & & & Incómodo & 7 & 1,02 & $\begin{array}{l}\text { “...es algo incómodo para los demás } \\
\text { compañeros” }\end{array}$ \\
\hline & & & Se exhiben & 7 & 1,02 & "se andan exhibiendo en todos lados..." \\
\hline & & & $\begin{array}{l}\text { Dan mal } \\
\text { ejemplo }\end{array}$ & 6 & 0,87 & $\begin{array}{l}\text { "...creen que es un mal ejemplo para el } \\
\text { resto de alumnos" }\end{array}$ \\
\hline & & & $\begin{array}{l}\text { Producen } \\
\text { miedo }\end{array}$ & 6 & 0,87 & “...por miedo a que algo pase ...” \\
\hline & & & Para cambiarles & 4 & 0,58 & "...para ver si así cambian de opinión” \\
\hline & & & $\begin{array}{l}\text { Considerado } \\
\text { enfermedad }\end{array}$ & 4 & 0,58 & “...piensan que... es una enfermedad..." \\
\hline & & & $\begin{array}{l}\text { Supuestos sin } \\
\text { derechos }\end{array}$ & 2 & 0,29 & "... creen que ellos no tienen derechos" \\
\hline
\end{tabular}


MASKANA, Vol. 2, No. 2, 2011

\begin{tabular}{|c|c|c|c|c|c|c|}
\hline \multirow{4}{*}{$\overbrace{0}^{\tilde{\sigma}}$} & \multirow{4}{*}{300} & \multirow{4}{*}{26,55} & $\begin{array}{l}\text { Son } \\
\text { compañeros y } \\
\text { estudiantes }\end{array}$ & 197 & 65,67 & $\begin{array}{l}\text { "...siguen siendo personas y miembros del } \\
\text { colegio..." }\end{array}$ \\
\hline & & & Lo ocultan & 11 & 3,67 & $\begin{array}{l}\text { "...de ese secreto saben amigos cercanos } \\
\text { o personas que comparten ... el mismo } \\
\text { secreto" }\end{array}$ \\
\hline & & & $\begin{array}{l}\text { Hay que } \\
\text { apoyarlos }\end{array}$ & 5 & 1,67 & $\begin{array}{l}\text { "...están en el mismo colegio y hay que } \\
\text { apoyarles" }\end{array}$ \\
\hline & & & Hay aceptación & 2 & 0,67 & $\begin{array}{l}\text { "Creo que la mentalidad se ha } \\
\text { cambiado...y ahora somos más abiertos" }\end{array}$ \\
\hline \multirow{3}{*}{$\cdot \frac{\dot{\sigma}}{\sum^{2}}$} & \multirow{3}{*}{27} & \multirow{3}{*}{2,39} & $\begin{array}{l}\text { Necesitan } \\
\text { apoyo }\end{array}$ & 2 & 7,41 & “... son personas que tienen problemas" \\
\hline & & & Son diferentes & 2 & 7,41 & "Porque les parece algo raro" \\
\hline & & & $\begin{array}{l}\text { Reciben } \\
\text { tratamientos }\end{array}$ & 2 & 7,41 & $\begin{array}{l}\text { "Les ayudan a mejorar...dan terapias y } \\
\text { mucho más" }\end{array}$ \\
\hline
\end{tabular}

En la sociedad

Las tendencias encontradas en nuestro estudio se repiten en el estudio realizado en España (Pichardo y col., 2009a\&b) y en Italia (Bertone \& Franchi, 2008); donde el 67,26\% de los adolescentes creen que en la sociedad, los LGBT son tratados de manera injusta en comparación a los otros. Los adolescentes que consideraron que son tratados injustamente, indicaron que es debido a que no son aceptados, por el contrario, son discrimidados y violentados, considerados anormales, pecadores, enfermos y que producen asco; además, se recalcó que no tienen los mismos derechos civiles en comparación a las parejas heterosexuales. Los que indicaron "de igual forma", rescataron que son seres humanos como el resto y que ahora son más visibles y aceptados; finalmente, los que señalaron que son mejor tratados dijeron que se debe a que reciben más apoyo.

Las adolescentes lesbianas respondieron a esta pregunta: $11(78,57 \%)$ de manera injusta, "Porque no son iguales según ellos (la sociedad) y que debe ser como esta dicho", $3(21,43 \%)$ de igual manera que al resto y $2(14,29 \%)$ de manera más favorable. Los adolescentes gays respondieron a esta pregunta, 10 (71,43\%) de manera injusta, "Criticarles, burlarse, tener repugnancia, la mayoría de personas hacen eso", 3 (21,43\%) de igual manera que al resto. Los y las adolescentes bisexuales respondieron a esta pregunta, $24(80 \%)$ de manera injusta, "Porque están cegados por una sola realidad que ha sido implantada en nuestra sociedad desde siempre", $5(16,67 \%)$ de igual manera que al resto y $1(3,33 \%)$ de manera más favorable. Entre los profesores, $25(80,65 \%)$ respondieron que son tratados injustamente "Nuestra sociedad es decir en nuestro país somos tradicionalistas, se nos es difícil aceptar abiertamente esta tendencia debido a nuestra formación", "Porque por lo general en nuestro país la homofobia, la discriminación a los gays, el aislamiento, la no aceptación de su condición y son injustamente tratados", 2 (6,45\%) como a los demás, ninguno señaló de manera más favorable. Nuevamente, adolescentes LGBT, profesores y el resto de participantes opinaron siguiendo la misma tendencia.

En suma, se pudieron detectar algunos de los componentes de las actitudes descritas por (Soriano, 1995). Se encontraron muchas características del componente negativo, en lo cognitivo (se considera LGBT como anormales o enfermos) y en lo afectivo (considerarlos productores de malestar y asco); mientras que, de la tendencia positiva se encontraron componentes conductuales y afectivos (personas normales y sujetos con derechos). 
Tabla 4. ¿Cómo crees que se trata a LGBT en la sociedad en comparación con los otros?

\begin{tabular}{|c|c|c|c|c|c|c|}
\hline & $\mathrm{N}$ & $\%$ & ¿Por qué? & $\mathrm{n}$ & $\%$ & Citas \\
\hline \multirow{8}{*}{ 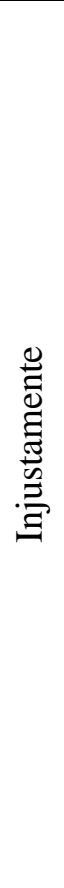 } & \multirow{8}{*}{760} & \multirow{8}{*}{67,26} & No aceptados & 183 & 24,08 & $\begin{array}{l}\text { "No toda la gente...está de acuerdo con } \\
\text { ese tema de preferencia sexual" }\end{array}$ \\
\hline & & & Discriminados & 155 & 20,39 & $\begin{array}{l}\text { "...la gente discrimina sin conocer a la } \\
\text { persona" }\end{array}$ \\
\hline & & & Violentados & 119 & 15,66 & $\begin{array}{l}\text { “...hay personas que los insultan, los } \\
\text { desprecian como si no fueran humanos, } \\
\text { son crueles" }\end{array}$ \\
\hline & & & $\begin{array}{l}\text { Considerado } \\
\text { anormal }\end{array}$ & 69 & 9,08 & $\begin{array}{l}\text { “... son diferentes y rompen con el patrón } \\
\text { normal, entonces la sociedad quiere que } \\
\text { encajen ..." }\end{array}$ \\
\hline & & & $\begin{array}{l}\text { Considerado } \\
\text { pecado }\end{array}$ & 44 & 5,79 & $\begin{array}{l}\text { "... la mayoría de personas ven a esto } \\
\text { como un pecado" }\end{array}$ \\
\hline & & & $\begin{array}{l}\text { No igualdad de } \\
\text { derechos }\end{array}$ & 26 & 3,42 & $\begin{array}{l}\text { "...les prohíben de ciertas cosas, como } \\
\text { casarse o adoptar un niño" }\end{array}$ \\
\hline & & & Producen asco & 20 & 2,63 & $\begin{array}{l}\text { “... a la mayoría de la sociedad les da } \\
\text { asco...” }\end{array}$ \\
\hline & & & $\begin{array}{l}\text { Considerado } \\
\text { enfermedad }\end{array}$ & 13 & 1,71 & $\begin{array}{l}\text { "La gente no tiene conocimiento y } \\
\text { piensan que ellos están enfermos" }\end{array}$ \\
\hline \multirow[t]{2}{*}{ 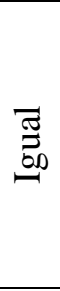 } & \multirow[t]{2}{*}{208} & \multirow[t]{2}{*}{18,41} & Son personas & 128 & 61,54 & $\begin{array}{l}\text { “... todos debemos tener los mismos } \\
\text { derechos y obligaciones así que sin } \\
\text { importar lo que somos todos somos } \\
\text { iguales" }\end{array}$ \\
\hline & & & $\begin{array}{l}\text { Ahora son más } \\
\text { visibles }\end{array}$ & 5 & 2,40 & $\begin{array}{l}\text { “...se vuelve algo más general las } \\
\text { personas ignoran esos hechos" }\end{array}$ \\
\hline$\frac{\overline{0}}{\sum^{\infty}}$ & 23 & 2,04 & $\begin{array}{l}\text { Necesitan más } \\
\text { apoyo }\end{array}$ & 8 & 34,78 & "para ayudarles más que a todos" \\
\hline
\end{tabular}

\subsection{Percepción de la reacción del otro si fueses un LGBT}

Al tratar de conocer cuál es la reacción que se esperaría del entorno ante la posibilidad de ser un LGBT, los adolescentes se expresan de la siguiente manera:

$\underline{\text { Familia }}$

El 46,55\% de adolescentes manifiestan que no saben cómo serían tratados dentro la familia. Los resultados encontrados en Cuenca difieren en distribución del estudio de España, pues, los españoles confían, en su mayoría, que recibirían el apoyo de su familia (Pichardo y col., 2009a\&b). Los adolescentes que piensan que su familia ejercería sobre ellos castigo físico, expresan que no serían aceptados por considerar anormal esta situación. Los que señalaron percibir rechazo, indicaron que se debe a que su familia lo considera algo anormal y pecado. Un grupo advirtió que se ignoraría el tema por ser una condición normal; sin embargo, algunos señalaron que sería una forma de esquivar el tema y una manera de manifestar su rechazo. Quienes esperarían apoyo, explicaron que esto se debería a que son miembros de la familia; y, los que señalaron que esperan que intenten cambiarlos, revelaron que es debido a que no serían aceptados.

Las adolescentes lesbianas respondieron $3(21,43 \%)$ me pegarían o darían una paliza, $4(28,57 \%)$ me rechazarían, "mi familia es demasiada cerrada", 2 (14,29\%) ignorarían el tema, 5 (35,71\%) no sé cómo reaccionarían, 2 (14,29\%) me apoyarían "Porque ellos me quieren", 4 (28,57\%) intentarían que cambiara. Los adolescentes gays respondieron $1(7,14 \%)$ me pegarían o darían una paliza, $2(14,29 \%)$ 
me rechazarían, 3 (21,43\%) ignorarían el tema, 6 (42,86\%) no sé cómo reaccionarían, 2 (14,29\%) intentarían que cambiara. Los y las adolescentes bisexuales respondieron $4(13,33 \%)$ me pegarían o darían una paliza, 10 (33,33\%) me rechazarían, 3 (10,00\%) ignorarían el tema, "Soy bisexual, al contarle a mi madre, lloró y de ahí hizo como si no le hubiera dicho nada, siempre intenta que salga con chicos y aún no comprende cómo pude elegir a una mujer como novia, pero tampoco me reprende", 11 (36,67\%) no sé cómo reaccionarían, 4 (13,33\%) me apoyarían, "Porque al comienzo sería difícil incluso para ellos, pero sé que al fin lo entenderían", 7 (23,33\%) intentarían que cambiara.

Tabla 5: ¿Si fueses un LGBT como piensas que te trataría tu familia?

\begin{tabular}{|c|c|c|c|c|c|c|}
\hline & $\mathrm{n}$ & $\%$ & ¿Por qué? & $\mathrm{n}$ & $\%$ & Citas \\
\hline \multirow{2}{*}{ 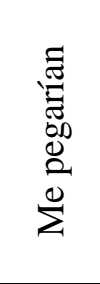 } & \multirow[b]{2}{*}{81} & \multirow[b]{2}{*}{7,17} & No lo aceptarían & 39 & 48,15 & $\begin{array}{l}\text { “... no les gustaría que su hijo sea } \\
\text { asi”" }\end{array}$ \\
\hline & & & $\begin{array}{l}\text { Considerado } \\
\text { anormal e } \\
\text { incorrecto }\end{array}$ & 19 & 23,46 & $\begin{array}{l}\text { “... piensa que es algo anormal, una } \\
\text { enfermedad o simplemente tal vez } \\
\text { ellos pensarían que el prestigio de la } \\
\text { familia...” }\end{array}$ \\
\hline \multirow{2}{*}{ 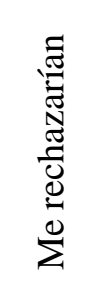 } & \multirow{2}{*}{142} & \multirow{2}{*}{12,57} & $\begin{array}{l}\text { Considerado } \\
\text { anormal e } \\
\text { incorrecto }\end{array}$ & 93 & 65,49 & “...piensan que es algo anormal...” \\
\hline & & & $\begin{array}{l}\text { Considerado } \\
\text { pecado }\end{array}$ & 11 & 7,75 & $\begin{array}{l}\text { “...mis papas son un poquito } \\
\text { anticuados y son religiosos y me } \\
\text { mandarían a un convento" }\end{array}$ \\
\hline \multirow{3}{*}{ 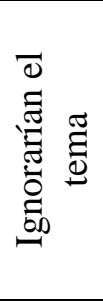 } & \multirow{3}{*}{63} & \multirow{3}{*}{5,58} & $\begin{array}{l}\text { Miembro de la } \\
\text { familia }\end{array}$ & 11 & 17,46 & $\begin{array}{l}\text { "Mi familia es bien abierta a } \\
\text { cualquier tema y eso no les } \\
\text { molestaría" }\end{array}$ \\
\hline & & & $\begin{array}{l}\text { Evitarían este } \\
\text { tema }\end{array}$ & 11 & 17,46 & $\begin{array}{l}\text { "Posiblemente se asustarían y } \\
\text { fingirían que no pasa nada" }\end{array}$ \\
\hline & & & No lo aceptarían & 10 & 15,87 & "No lo aceptarían" \\
\hline$z_{z}$ & 526 & 46,55 & & & & \\
\hline 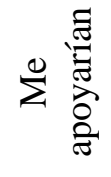 & 145 & 12,83 & $\begin{array}{l}\text { Miembro de la } \\
\text { familia }\end{array}$ & 126 & 86,90 & $\begin{array}{l}\text { "Me apoyarian porque me quieren } \\
\text { demasiado como para no apoyarme" }\end{array}$ \\
\hline \multirow{2}{*}{ 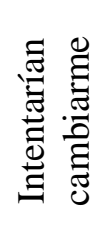 } & \multirow[t]{2}{*}{241} & \multirow[t]{2}{*}{21,33} & No lo aceptarían & 117 & 48,55 & $\begin{array}{l}\text { "... a mi familia no les gusta eso para } \\
\text { nada me matarían, pucha eso sería } \\
\text { demasiado poco para todo eso" }\end{array}$ \\
\hline & & & Para ayudarme & 77 & 31,95 & $\begin{array}{l}\text { "...cuando estoy en mal camino tratan de } \\
\text { apoyarme" }\end{array}$ \\
\hline
\end{tabular}

$\underline{\text { Amistades }}$

En relación con la percepción de lo que harían sus amigos, la mayoría, el 36,64\% de los adolescentes, no puede imaginar la reacción de ellos, coincidiendo en tendencia con el estudio realizado en España (Pichardo y col., 2009a\&b). En este estudio, el 33,45\% expresó que esperarían rechazo de ellos. Quienes esperarían agresión física o rechazo indicaron que se debe a que este tema no es aceptado o entendido, resulta incómodo, y puede generar burlas, miedo y asco. Entre los que esperan que se ignore el tema se encontró que este podría ser considerado normal; sin embargo, otros pensaron que 
sería una manera de esquivar un tema incómodo. Los que esperaron apoyo rescataron que es debido al valor de las amistades que tienen; finalmente, los que esperan que los presionen a cambiar indicaron que es porque se lo considera anormal o es para ayudarlos.

Tabla 6: ¿Si fueses LGBT cómo piensas que te tratarían tus amigos?

\begin{tabular}{|c|c|c|c|c|c|c|}
\hline & $\mathrm{n}$ & $\%$ & ¿Por qué? & $\mathrm{n}$ & $\%$ & Citas \\
\hline 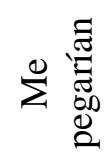 & 35 & 3,10 & $\begin{array}{l}\text { Considerado } \\
\text { anormal e } \\
\text { incorrecto }\end{array}$ & 17 & 48,57 & $\begin{array}{l}\text { "...lo tomarían como algo } \\
\text { inadecuado" }\end{array}$ \\
\hline \multirow{5}{*}{ 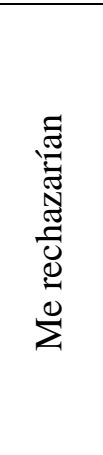 } & \multirow{5}{*}{378} & \multirow{5}{*}{33,45} & $\begin{array}{l}\text { Considerado } \\
\text { anormal e } \\
\text { incorrecto }\end{array}$ & 233 & 61,64 & $\begin{array}{l}\text { "... es algo diferente para ellos y te } \\
\text { rechazarian" }\end{array}$ \\
\hline & & & Por incomodidad & 29 & 7,67 & $\begin{array}{l}\text { "Mis amigas se sentirían } \\
\text { incómodas..." }\end{array}$ \\
\hline & & & Se burlarían & 16 & 4,23 & “... se burlarian y molestarian” \\
\hline & & & Producen miedo & 4 & 1,06 & $\begin{array}{l}\text { "...tuvieran miedo de que les pueda } \\
\text { hacer algo" }\end{array}$ \\
\hline & & & Producen asco & 2 & 0,53 & "dan asco" \\
\hline \multirow{2}{*}{ 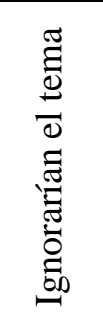 } & \multirow[t]{2}{*}{61} & \multirow[t]{2}{*}{5,40} & Es normal & 20 & 32,79 & $\begin{array}{l}\text { "A ellos les daría igual, porque ya } \\
\text { están acostumbrados a relacionarse } \\
\text { con esa clase de gente, algunos son } \\
\text { gays, lesbianas, entonces no les } \\
\text { prestarían mucha atención al tema" }\end{array}$ \\
\hline & & & $\begin{array}{l}\text { No sabrían que } \\
\text { hacer }\end{array}$ & 16 & 26,23 & $\begin{array}{l}\text { "Lo ignorarían, para no sentirse } \\
\text { incómodos" }\end{array}$ \\
\hline$z_{z} \ddot{n}$ & 414 & 36,64 & & & & \\
\hline 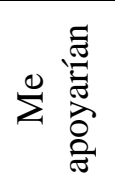 & 115 & 10,18 & Son mis amigos & 81 & 70,43 & $\begin{array}{l}\text { “...me apoyarían porque por esa } \\
\text { razón son mis amigos, tienen una } \\
\text { mentalidad abierta y no me } \\
\text { juzgarían” }\end{array}$ \\
\hline \multirow{2}{*}{ 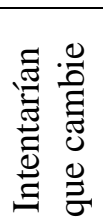 } & \multirow[b]{2}{*}{127} & \multirow[b]{2}{*}{11,24} & $\begin{array}{l}\text { Considerado } \\
\text { anormal }\end{array}$ & 50 & 39,37 & $\begin{array}{l}\text { "...eso no le pareciera normal a los } \\
\text { que me rodean" }\end{array}$ \\
\hline & & & Para ayudarme & 20 & 15,75 & $\begin{array}{l}\text { “...los buenos amig@s darian todo } \\
\text { por ti para cambiar hasta lograrlo" }\end{array}$ \\
\hline
\end{tabular}

Las adolescentes lesbianas respondieron a esta pregunta: $2(14,29 \%)$ me pegarían o darían una paliza, $7(50 \%)$ me rechazarían, $4(28,57 \%)$ ignorarían el tema, $3(21,43 \%)$ no sé cómo reaccionarían, $4(28,57 \%)$ me apoyarían, $1(7,14 \%)$ intentarían que cambiara. Los adolescentes gays respondieron, 1 (7,14\%) me pegarían o darían una paliza, $6(42,86 \%)$ me rechazarían, $1(7,14 \%)$ ignorarían el tema, 5 $(35,71 \%)$ no sé cómo reaccionarían, $2(14,29 \%)$ me apoyarían, $2(14,29 \%)$ intentarían que cambiara. Los y las adolescentes bisexuales respondieron $2(6,67 \%)$ me pegarían o darían una paliza, $9(30 \%)$ me rechazarían, 2 (6,67\%) ignorarían el tema, 10 (33,33\%) no sé cómo reaccionarían, 10 (33,33\%) me apoyarían, "Cuando se lo conté a mi grupo verdadero de amigos, y ellos(as) me apoyaron por completo, eso me mostró quienes son amigos y quienes no", 2 (6,67\%) intentarían que cambiara. Un adolescente bisexual señaló que recibiría castigo físico y rechazo, "Basado en experiencia yo sé porque lo hicieron, aparentemente son egoístas y homofóbicos". 


\section{Profesores}

Los adolescentes cuencanos, en su mayoría, no sabrían que esperar de sus profesores, 41,59\%; otro grupo cree que se ignoraría el tema, 20,71\%; a diferencia de los españoles que perciben como generalidad que ellos ignorarían el tema (Pichardo y col., 2009a\&b).

Quienes esperaban recibir rechazo pensaron que es por considerarlo anormal o por no saber cómo proceder. Entre los que esperan que se ignore el tema, se manifestó que los profesores se mantienen al margen de temas personales de los estudiantes y enfatizan solo lo académico; además, otros indicaron que sería una forma de apoyo o de mantenerse neutrales, o simplemente por no saber qué hacer ante la situación. Los que expresaron que los apoyarían, indicaron que sería para respaldarlos; aunque, también se consideró como "ayuda" el tratar de cambiarlos. Finalmente, los que piensan que los obligarían a cambiar señalaron como razones que no los entenderían, es más, que lo han observado ya; además, surgió nuevamente la idea de que cambiarlos representa una forma de apoyarlos.

Tabla 7: ¿Si fueses LGBT cómo piensas que te tratarían tus profesores?

\begin{tabular}{|c|c|c|c|c|c|c|}
\hline & $\mathrm{n}$ & $\%$ & ¿Por qué? & $\mathrm{n}$ & $\%$ & Citas \\
\hline 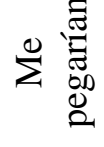 & 7 & 0,62 & No lo aceptarían & 4 & 57,14 & "No son aceptados" \\
\hline \multirow{2}{*}{ 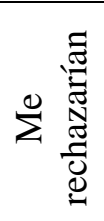 } & \multirow[b]{2}{*}{68} & \multirow[b]{2}{*}{6,02} & $\begin{array}{l}\text { Considerado anormal e } \\
\text { incorrecto }\end{array}$ & 19 & 27,94 & $\begin{array}{l}\text { "... muchos profes piensan que es } \\
\text { algo malo" }\end{array}$ \\
\hline & & & No sabrían que hacer & 7 & 10,29 & $\begin{array}{l}\text { "No saben como tratar a esas } \\
\text { personas" }\end{array}$ \\
\hline \multirow{4}{*}{ 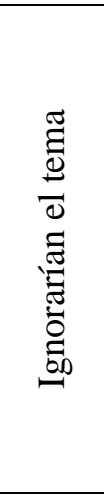 } & \multirow{4}{*}{234} & \multirow{4}{*}{20,71} & No es su problema & 116 & 49,57 & $\begin{array}{l}\text { "...ellos son profesores y no tienen } \\
\text { por qué importarles la inclinación } \\
\text { sexual de cada alumno solo deben } \\
\text { dedicarse a enseñar" }\end{array}$ \\
\hline & & & Para apoyarme & 19 & 8,12 & $\begin{array}{l}\text { "Para no hacerme sentir mal y que } \\
\text { mis compañeras me molesten" }\end{array}$ \\
\hline & & & No sabrían que hacer & 16 & 6,84 & “...se sentirían incómodos" \\
\hline & & & Mantenerse neutrales & 13 & 5,56 & $\begin{array}{l}\text { “...los profesores deberían } \\
\text { mantenerse neutros a ese tipo de } \\
\text { situaciones" }\end{array}$ \\
\hline$z: \ddot{n}$ & 470 & 41,59 & & & & \\
\hline \multirow{2}{*}{ 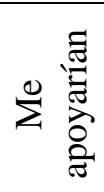 } & \multirow[t]{2}{*}{168} & \multirow[t]{2}{*}{14,87} & Para respaldarlos & 120 & 71,43 & $\begin{array}{l}\text { "...para eso están en el colegio } \\
\text { para aconsejar y no para juzgar" }\end{array}$ \\
\hline & & & Para que cambie & 2 & 1,19 & "Me apoyarían para no ser gay" \\
\hline \multirow{3}{*}{ 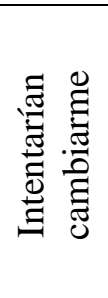 } & \multirow{3}{*}{153} & \multirow{3}{*}{13,54} & No lo entenderían & 51 & 33,33 & $\begin{array}{l}\text { "...pensarian que es una etapa y } \\
\text { que estoy confundida”" }\end{array}$ \\
\hline & & & Para ayudarme & 46 & 30,07 & $\begin{array}{l}\text { “...los profesores siempre están } \\
\text { para ayudar a sus estudiantes” }\end{array}$ \\
\hline & & & Lo he visto & 1 & 0,65 & $\begin{array}{l}\text { "...y a a pasado antes en el } \\
\text { colegio" }\end{array}$ \\
\hline
\end{tabular}


Las adolescentes lesbianas respondieron $1(7,14 \%)$ me pegarían o darían una paliza, $2(14,29 \%)$ me rechazarían, $3(21,43 \%)$ ignorarían el tema, $4(28,57 \%)$ no sé cómo reaccionarían, $6(42,86 \%)$ me apoyarían, 2 (14,29\%) intentarían que cambiara. Los adolescentes gays 1 (7,14\%) me rechazarían, 2 $(14,29 \%)$ ignorarían el tema, $5(35,71 \%)$ no sé cómo reaccionarían, $2(14,29 \%)$ me apoyarían, 4 $(28,57 \%)$ intentarían que cambiara. Los y las adolescentes bisexuales respondieron $1(3,33 \%)$ me pegarían o darían una paliza, $4(13,33 \%)$ me rechazarían, "porque me odiarían", $4(13,33 \%)$ ignorarían el tema, $16(53,33 \%)$ no sé cómo reaccionarían, 5 (16,67\%) me apoyarían, $3(10 \%)$ intentarían que cambie.

\section{Compañeros}

Los adolescentes cuencanos respondieron en su mayoría $(32,65 \%)$ que esperarían rechazo de sus compañeros, los resultados encontrados son similares a los del estudio español (Pichardo y col., 2009a\&b).

Los adolescentes indican que de reconocerse LGBT, sus compañeros les agrederían físicamente; quienes manifestaron que los rechazarían, señalaron que se debería a que no los aceptarían, por considerarlos anormales, harían burla de esto, sería una situación incómoda, no lo entenderían, o para tratar de hacerlos cambiar; expresan que esta situación ha sido vivenciada ya, en el colegio. Quienes esperan que este tema sea ignorado, indicaron que al ser un tema normal no tiene por qué generar mayor polémica; no obstante, hay quienes alegaron que sería para evitar el tema o porque generalmente esta condición se mantiene oculta. Los adolescentes que consideraron esperar apoyo, indicaron que al ser sus compañeros, no se puede esperar menos de ellos; aunque, otros manifestaron que el apoyo sería para cambiarlos. Finalmente, entre los que señalaron que apoyarlos significa intentar cambiarlos, unos indicaron que esto sería por su bien; mientras otros respondieron que es porque no los aceptarían.

Las adolescentes lesbianas respondieron $2(14,29 \%)$ me pegarían o darían una paliza, $6(42,86 \%)$ me rechazarían, $1(7,14 \%)$ ignorarían el tema, $4(28,57 \%)$ no sé cómo reaccionarían, $3(21,43 \%)$ me apoyarían, $2(14,29 \%)$ intentarían que cambiara. Los adolescentes gays respondieron $2(14,29 \%)$ me pegarían o darían una paliza, $8(57,14 \%)$ me rechazarían, "no tendría amigos, seria aislado", 1 $(7,14 \%)$ ignorarían el tema, 4 (28,57\%) no sé cómo reaccionarían, 1 (7,14\%) me apoyarían.

Los y las adolescentes bisexuales respondieron $2(6,67 \%)$ me pegarían o darían una paliza, 11 $(36,67 \%)$ me rechazarían, $5(16,67 \%)$ ignorarían el tema "Se sienten incómodos, por lo tanto prefieren ignorar", $13(43,33 \%)$ no sé cómo reaccionarían, 1 (3,33\%) me apoyarían, 2 (6,67\%) intentarían que cambiara.

A manera general podemos observar que si bien se considera incorrecto el maltrato hacia ellos, existe la percepción por parte de los adolescentes de un ambiente hostil hacia los LGBT, y se puede reconocer que estas personas están estigmatizadas por una cantidad de prejuicios en torno a ellos, prejuicios que son compartidos y asumidos por los adolescentes, como el de considerarlos peligrosos, antinaturales. Además, los mismos LGBT perciben que son estigmatizados y muchos de ellos lo aceptan como algo natural; esto se ve reflejado en el miedo a las reacciones de los demás, incluyendo a su familia y compañeros, configurando las bases para el desarrollo de una autohomofobia y de diversas reacciones emocionales que pueden llegar a motivar ideas y actos autodestructivos, como lo afirma (Ortiz, 2005).

Tabla 8: ¿Si fueses LGBT, cómo piensas que te tratarían tus compañeros?

\begin{tabular}{|c|c|c|c|c|c|c|}
\hline & $\mathrm{N}$ & $\%$ & ¿Por qué? & $\mathrm{n}$ & $\%$ & Citas \\
\hline 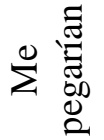 & 27 & 2,39 & No lo aceptarían & 13 & 48,15 & "No comprenden eso" \\
\hline
\end{tabular}


MASKANA, Vol. 2, No. 2, 2011

\begin{tabular}{|c|c|c|c|c|c|c|}
\hline \multirow{7}{*}{ 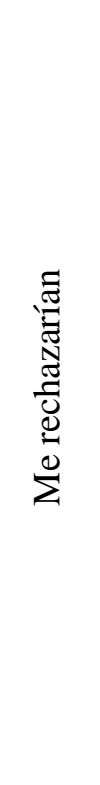 } & \multirow{7}{*}{369} & \multirow{7}{*}{32,65} & No lo aceptarían & 106 & 28,73 & $\begin{array}{l}\text { "...es complicado que me } \\
\text { aceptaran" }\end{array}$ \\
\hline & & & $\begin{array}{l}\text { Considerado anormal } \\
\text { e incorrecto }\end{array}$ & 60 & 16,26 & $\begin{array}{l}\text { “...piensan que está mal o es } \\
\text { incorrecto ser gay...” }\end{array}$ \\
\hline & & & Se burlarían & 27 & 7,32 & $\begin{array}{l}\text { “...son tema de burla para muchos } \\
\text { jóvenes" }\end{array}$ \\
\hline & & & Situación incómoda & 18 & 4,88 & $\begin{array}{l}\text { "Se sentirian un poco incomodo } \\
\text { que alguien le guste de su mismo } \\
\text { sexo" }\end{array}$ \\
\hline & & & No lo entenderían & 17 & 4,61 & $\begin{array}{l}\text { "...la educación es escaza y la } \\
\text { edad también influye" }\end{array}$ \\
\hline & & & Lo he visto & 7 & 1,90 & $\begin{array}{l}\text { “...en el colegio hay un chico gay y } \\
\text { todos le rechazan entonces también } \\
\text { creo que me rechazarian” }\end{array}$ \\
\hline & & & Para que cambie & 4 & 1,08 & $\begin{array}{l}\text { "Eso no es lo correcto y me } \\
\text { apoyarían para que cambie" }\end{array}$ \\
\hline \multirow{3}{*}{ 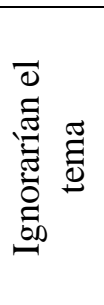 } & \multirow{3}{*}{81} & \multirow{3}{*}{7,17} & Un compañero más & 24 & 29,63 & $\begin{array}{l}\text { "... a ellos no les importa o no les } \\
\text { debería importar la vida de las } \\
\text { estudiantes" }\end{array}$ \\
\hline & & & Tema incómodo & 15 & 18,52 & $\begin{array}{l}\text { “...se sentirían incómodos como } \\
\text { todos nos podremos sentir...” }\end{array}$ \\
\hline & & & No contaría & 2 & 2,47 & "A veces no es necesario contarlo" \\
\hline$\stackrel{0}{z}$ & 456 & 40,35 & No se & & & \\
\hline \multirow{2}{*}{ 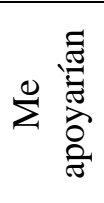 } & \multirow[b]{2}{*}{54} & \multirow[b]{2}{*}{4,78} & Son mis compañeros & 27 & 50,00 & "Tengo buenos compañeros" \\
\hline & & & Apoyo para cambiar & 1 & 1,85 & "Para cambiar" \\
\hline \multirow{2}{*}{ 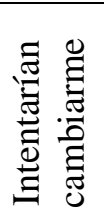 } & \multirow[t]{2}{*}{97} & \multirow[t]{2}{*}{8,58} & Para ayudarme & 32 & 32,99 & $\begin{array}{l}\text { "...son magníficos compañeros } \\
\text { hicieran lo que sea para que yo } \\
\text { pueda cambiar" }\end{array}$ \\
\hline & & & No es aceptado & 20 & 20,62 & "... quisieran que fuera normal" \\
\hline
\end{tabular}

\section{CONCLUSIONES}

La mayoría de los adolescentes consideran que no se debe despreciar a los LGBT, por reconocerlos ante todo como seres humanos; pese a esto, un grupo minoritario tiende a justificar un posible maltrato hacia ellos, debido a la presencia de mitos que los catalogan como anormales, enfermos, pecadores, etc. Los adolescentes perciben, en su mayoría, como espacios de intolerancia e injusticia para los LGBT, la familia, el colegio y la sociedad en general. De estos espacios, es la familia donde se percibe menos discriminación en el trato, y es en la sociedad donde se piensa encontrar el peor trato. Al indagar sobre las expectativas de la respuesta esperada en la familia, amistades, profesores y compañeros ante una hipotética orientación LGBT, la mayoría no sabría que esperar. Sin embargo, la reacción esperada desde los amigos y compañeros es el rechazo debido a que no se aceptaría su orientación. Desde los padres, se esperaría que se los trate de cambiar y de los profesores que no se involucren en el tema. Otro hallazgo es que, la mayoría, considera que la falta de aceptación que se 
espera es debida a que es un tema tratado incorrectamente y que aún se mantienen muchos prejuicios que estigmatizan a este grupo.

De otra parte, existió un considerable número de participantes que mantuvieron ellos mismos creencias prejuiciosas hacia LGBT, considerando aceptable que se los violente o se los trate de rehabilitar en sus preferencias sexuales. Llamativamente, se encontró igual tendencia de respuestas entre los adolescentes LGBT y heterosexuales, situación que estaría relacionada con la influencia cultural que modela por igual las actitudes de las personas, con independencia de su orientación e intereses sexuales. De todo esto, resulta imperativa la necesidad de analizar este tema con adolescentes y profesores para eliminar mitos y prejuicios hacia esta minoría. Además, de incorporar contenidos educativos que promuevan la igualdad y el respeto hacia los LGBT. Este proceso educativo debería involucrar a docentes y padres de familia para que el tema sea visibilizado en procura de modificar los patrones cognitivos y actitudinales detectados.

\section{AGRADECIMIENTOS}

Agradecemos a los miembros de la Dirección de Investigación de la Universidad de Cuenca por brindarnos el apoyo financiero y técnico para la investigación, a los colegios de la ciudad por permitirnos realizar las encuestas. A los estudiantes Marco Ambrosi, Felipe Cáceres y Christian Pacheco que participaron como ayudantes de investigación de las Facultades de Filosofía, Letras y Ciencias de la Educación; Psicología y Ciencias Médicas, respectivamente. Además, a Daysi Rivera y Wilson Rocano por el apoyo para la triangulación de la información.

\section{BIBLIOGRAFÍA}

Ardila, R., 2002. Homosexualidad y Psicología. Manual Moderna, Bogotá, Colombia, 1-3.

Antezana, M., 2007. Homosexualidad, familia y apoyo social. Gac. Med. Bol. , 30(1).

Baron, R., 1997. Fundamentos de psicología (3ra Ed.). Prentice Hall, México D.F.

Bertone, C., M. Franchi, 2008. Las experiencias familiares de jóvenes lesbianas y gays en Italia. Actas de conferencias: Family matters - Prevenir la violencia homofóbica contra las personas jóvenes gays y lesbianas a través del apoyo de sus familias. Florencia, Italia, 6-36.

Briñol, P., L. De la Corte, A. Becerra, 2001. ¿Qué es persuación? Biblioteca Nueva, Madrid, España.

Creswell, J., 2008. Research design (3ra Ed.). Saga Publications Inc., Nebraska, USA, 296 págs.

Crooks, R., K. Baur, 2009. Nuestra sexualidad (10e Ed.). Cengage Learning Ed., México D.F., México, 260-292.

Dovido, J., B. Major, J. Crocker, 2003. Stigma: Introduction and overview. En: Heatherton, T., R. Kleck, M. Hebl, J. Hull (Eds.). The social psychology of stigma. The Guilford Press, New York, USA, 30 págs.

Goffman, E., 1995. Estigma. La identidad deteriorada. Amorrortu Editores, Buenos Aires, Argentina.

González, E., V. Martínez, C. Leyton, A. Bardi, 2004. Orientación sexual: Un desafío actual para la atención de adolescentes. Rev. Sogia., 11(3), 69-78.

Graglia, M., R. Lelleri, L.Pietrantoni, 2008. Modos de divulgación social. Actas de conferencias: Family matters - Prevenir la violencia homofóbica contra las personas gays y lesbianas a través del apoyo de sus familias. Florencia, Italia, 41-43 págs.

Hernández, R., C. Fernández, P. Baptista, 2007. Metodología de la investigación (4ta Ed.). McGrawHill, México D.F, México, 45-805. 
Martínez, J., R. Sampedro, 2003. La percepción social. En: Rodríguez, C., Psicología social: Cómo influimos en el pensamiento y la conducta de los demás. Biblioteca Universitaria, Madrid, España, 67-80.

Ortiz, L, 2005. Influencia de la opresión internalizada sobre la salud mental de bisexuales, lesbianas y homosexuales en la ciudad de México. Salud mental, 4(28), 49-65

Papalia, D., S. Wendkos, R. Duskin, 2005. Psicología del desarrollo de la infancia a la adolescencia (9na Ed.). McGraw-Hill, México D.F., México.

Pichardo, J.I., B. Molinuevo, P. Rodriguez, N. Martín, M. Romero, 2009a. Actitudes hacia la diversidad sexual de la población adolescente de Coslada (Madrid) y San Bartolomé de Tirajana. Descargado de http:www.educarenigualdad.org/upload/Doc_237_actitudes_diversidad_ sexual.pdf, 75 págs, el 15 de mayo de 2010.

Pichardo, J.I., B. Molinuevo, P. Rodriguez, N. Martín, M. Romero, 2009b. Adolescentes ante la diversidad sexual. Homofobia en los centros educativos. Los Libros de la Catarata, Madrid, España, 158 págs.

Pinos, V., G. Pinos, 2011. Actitudes de los adolescentes escolarizados de Cuenca hacia la diversidad sexual. Maskana, 2(1), 1-16.

Piñuel, J., 2002. Epistemología, metodología y técnica del análisis de contenido. Estudios de Sociolinguística, 3(1), 1-42.

Santrock, J, 2004. Adolescencia: psicología del desarrollo (9na Ed.). McGraw-Hill, México D.F., México, 298-302.

Shibley, J., J. Delamater, 2006. Sexualidad Humana (9na Ed.). McGraw-Hill, México D.F., México, 359-387.

Soriano, E, 1995. Estado actual de la investigación sobre la homofobia. Estudios de Psicología, 54, 59-72.

Worchel, S., J. Cooper, G. Goethals, J. Olson, 2003. Psicología social. Thompson, Madrid, España, 349-361. 